\title{
De las Drip-Paintings de Jackson Pollock a la Forma Abierta de Earle Brown: Interconexiones metodológicas en los años de la Escuela de Nueva York
}

\author{
From the Drip-Paintings of Jackson Pollock to the Open Form of Earle \\ Brown: Methodological Interconnections in the Years of the New York \\ School
}

\author{
Íñigo Sarriugarte Gómez \\ Universidad del País Vasco, UPV-EHU \\ https://orcid.org/0000-0003-0206-9864 \\ inigo.sarriugarte@ehu.eus
}

Recibido: 20/09/2021; Revisado: 23/12/2021; Aceptado: 29/12/2021

\section{Resumen}

A partir de la Segunda Guerra Mundial, se fue gestando el nacimiento de la Escuela de Nueva York, donde una serie de músicos y pintores empezaron a desarrollar nuevas pautas experimentales, lo que facilitaría el surgimiento del Expresionismo Abstracto. Earle Brown (1926-2002), partiendo del análisis del dripping en las pinturas de Jackson Pollock en base a la espontaneidad, lo aleatorio y la libertad de ejecución e interpretación diversificada, desarrolló la forma abierta en las pautas musicales, donde se facilitaba la libertad del intérprete en el momento de la ejecución musical y se articulaban propuestas cargadas de una multiplicidad de ejecuciones diversas.

Palabras clave: Pollock, Brown, Dripping, Escuela de Nueva York, Forma Abierta.

\begin{abstract}
The end of World War II ushered in the gradual emergence of the New York School, where a number of musicians and painters began to develop new experimental approaches, leading to the advent of Abstract Expressionism. Taking his lead from an analysis of spontaneity, randomness, freedom of execution and interpretative diversity observed in the dripping of Jackson Pollock's paintings, Earle
\end{abstract}


Brown (1926-2002) developed the open form of musical composition and notation, where the musician was given freedom of interpretation at the time of musical performance and the score itself proposed a multitude of different ways for the piece to be performed.

Keywords: Pollock, Brown, Dripping, New York School, Open Form.

\section{BREVE INTRODUCCIÓN A LA RELACIÓN PINTURA-MÚSICA DESDE LAS VANGUARDIAS HISTÓRICAS}

Las teorizaciones conductivas entre los lenguajes musicales y plásticos han sido una práctica anidada en numerosos momentos durante la historia del arte, resultando uno de los episodios de mayor conexión experimental la que se produce a partir del surgimiento de las Vanguardias Históricas. En cualquier caso, esta orientación conectiva, no evita ciertas dificultades, tal y como explica Román DE LA CALLE (2007:91):

El que las confrontaciones entre música y pintura no hayan sido ni mucho menos extrañas, a lo largo de la historia, y siga siendo aún una especie de reto recurrente, mantenido en el contexto de la cultura, el hecho de seguir intentándolo, una y otra vez, hace evidente que esa arriesgada operación de instaurar juegos de contrastes entre música y artes plásticas no deja de ser, cuando menos, escabrosa y complicada.

Entre las diferentes reflexiones que se han ido entretejiendo entre ambos campos culturales, debemos anotar los trabajos teóricos del pensador alemán Theodor Adorno, quien establecía una semejanza correlativa entre la pintura contemporánea y la música en cuanto al objeto, entendiendo esta cuestión desde el instante en que la práctica pictórica había desechado asemejarse con el objeto que utilizaba como base para el desarrollo del arte pictórico; de igual manera la práctica musical decide renunciar al orden tonal. Por lo tanto, para ADORNO (2003: 41), una vez desprendido de la atadura entre objeto y arte, se genera un fenómeno de desobediencia ante la realidad preexistente, lo que invitaba tanto en el ámbito pictórico como musical a todo tipo de experimentaciones. De hecho, este filósofo germano llegó a establecer que la música lejos de inspirarse por la pintura tendía más bien a imitar su composición estructural. Incluso, se puede ir más allá, tal y como lo analiza Lorena VALDEBENITO (2011: 54), «da paso esto a un momento distinto en la música, donde el sujeto vuelve a tomar un papel secundario, y la cosa un papel protagónico, donde el sujeto que antes creador sólo es intérprete de los requerimientos de la cosa».

Si trazamos un somero recorrido, sin asumir una exhaustividad detallada, de estas vinculaciones interdisciplinares desde la aparición de las Vanguardias Históricas a finales del siglo XIX, deberíamos citar las primeras experiencias musicales realizadas por parte de Debussy en relación con el impresionismo. Igualmente, desde una aportación experimental, resultan destacables los diferentes planteamientos generados dentro del expresionismo con el dodecafonismo de Arnold Schoenberg, y los demás miembros de la Escuela de Viena, como Schumann, Brahms y Mahler, seguido por otras pautas sonoras no menos interesantes generadas por el compositor futurista Francesco Balilla Pratella, que 
animado por Marinetti, redacta en 1910 el histórico Manifiesto de Música Futurista, quien a su vez llamaría el interés de Luigi Russolo, lo que generaría la redacción de una carta con el manifiesto denominado El arte de los Ruidos (1913).

En un acercamiento a las especulaciones rupturistas del mundo dada, resulta célebre el siguiente episodio cuando Marcel Duchamp y sus dos hermanas extrajeron las notas de la escala al azar de un sombrero, tildando a la composición resultante Musical Erratum (1913), que sería denominado posteriormente por John Cage como «una simple pero interesante manera de trabajar» (CAGE, 1990: 50-51). Marcel Duchamp había comentado este evento al crítico de arte Calvin Tomkins (1968: 33) de la siguiente manera: «Tu casualidad no es la misma que la mía, al igual que cuando tiras los dados rara vez será la misma tirada que la mía». En el dadaísmo, también deberíamos citar los trabajos de Erwin Schulhoff, amigo del pintor George Grosz, a quien dedicó Suite for Chamber Orchestra (1921). Por otro lado, su obra anterior In futurum (1919) quedaba escrita en compases absurdos, siendo compuesta tan solo de pausas y con la indicación de que debía ser tocada «tutto il canzone con espressione e sentimento ad libitum, sempre, sin al fine!» (Schulhoff, en Kim-CoHEN, 2009: 18). También, la provocación quedaría reflejada en su sátira musical contra el militarismo alemán titulada Sinfonía Germánica (1919) en tres movimientos, compuesta con una serie de disonantes vocales conjuntadas con instrumentos desafinados que difícilmente podían entonar el himno nacional alemán.

Junto a estos anteriores ejemplos, deberíamos adjuntar las experimentaciones del grupo Les Six, compuesto entre otros por Georges Auric, Louis Durey, Arthur Honegger y Francis Poulenc, quienes se moverían entre el dadaísmo y el surrealismo durante las primeras décadas del siglo xx. Theodor AdORNO (2002: 409) cita también como composiciones cercanamente surrealistas la ópera Auge y caída de la ciudad de Mahagonny (1930), con música de Kurt Weill y libreto en alemán de Bertolt Brecht, junto con otras obras procedentes de Igor Stravinsky, en particular L'Histoire du soldat (1917). En esta misma línea de citaciones históricas, tampoco, deberíamos olvidar los nombres de André Souris y Pierre Schaeffer.

Para el pintor suizo Paul Klee, los principales paralelismos que se podían establecer entre pintura y música remitían a los aspectos estructurales. Cuando Paul KLEE (1987: 28) extrapolaba la máxima: «Sólo me alegraba lo prohibido», recordando lo que marcaba su procedimiento táctico, este mismo parangón se podía apreciar en Pierre Boulez (1984: 452) al comentar: «Lo que busco, desde que he cobrado conciencia de que existe un muro -o más bien una serie de muros- es hacer caer, de alguna forma, esos muros. De hecho, el factor tabicamiento es para mí la muerte de las cosas. Para ser eficaz, todo debería interpenetrarse».

Simplemente con el objetivo de acotar y finalizar esta introducción, igualmente resultan de interés las extrapolaciones pictóricas y escenográficas del pintor ruso Nicolás Roerich en base al ballet y concierto orquestal de Le Sacre du printemps (1913) de Stravinsky, así como las correspondencias sinestésicas entre música y pintura, que abordaron Kandinsky, Kupka o Mondrian, siendo en el caso del pintor neoplasticista bajo la forma moderna del jazz. 


\section{LA ESCUELA DE NUEVA YORK EN EL CONTEXTO DE LOS AÑOS 40 Y 50}

La sensación general después de la Segunda Guerra Mundial se caracterizaba por un visible pesimismo ante lo que suponía en cierta manera el fracaso de los valores ideológicos de la Ilustración, lo que impulsara al artista a cerrarse en sí mismo, retomar su interior y refugiarse en su propia subjetividad sartriana y en una especie de angustia camusiana, siendo la única salida la exteriorización de su propia individualidad. No obstante, el ambiente cultural de la posguerra se vio favorecido por la propia Administración norteamericana al poner en marcha un Plan Marshall cultural, que se desarrollaría de manera lineal y paralelo al gestado en el ámbito económico y social. Este plan trataba de incentivar relaciones culturales entre los diferentes países que habían sido aliados, a la vez que pretendía instalar en la Alemania de posguerra una renovada versión cultural que la acercara a los parámetros de los países vencedores, recuperando y promoviendo festivales musicales, como los de Donaueschingen y Darmstadt.

Esta coyuntura geocultural no siempre generó las dosis de armonía ilustrada que se pretendía, de hecho, es bien conocida la dialéctica teórica y musical tanto a un lado como al otro del Atlántico, tal y como sucedió entre Nono y John Cage, cuando este primero con su ensayo, presentado en 1959 en el encuentro musical IFNM, reprendía las conferencias dadas en 1958 por John Cage, cambiando su énfasis sobre la indeterminación y criticando la asimilación de estas ideas por parte de Stockhausen al anotar lo siguiente: «Hoy estamos persuadidos en hacer creer que la improvisación es una liberación, una garantía de la libertad de uno mismo. Y, por otro lado, por supuesto, este orden está limitado, es la contención de uno mismo» (Nono, en BEAL, 2006: 113). ${ }^{1}$

Todo se encaminaba a colocar la cultura moderna norteamericana en primera fila y de hecho ya en 1948 Clement Greenberg decidió en enero de ese mismo año proclamar que el arte norteamericano era el primero del mundo, apareciendo dichas declaraciones en una serie de artículos en las revistas Partisan Review y Nation, donde se examinaba el arte norteamericano. El objetivo de estas manifestaciones de Greenberg era relanzar la idea de «independencia cultural», mostrando la existencia de una autonomía cultural respecto a la europea y sobre todo respecto a la parisina. De este modo, Clement Greenberg se convertiría en el paladín y defensor a ultranza de la supremacía del arte norteamericano frente al europeo, afirmando que el arte de Nueva York y Jackson Pollock ocupaban los lugares principales en la esfera artística del momento. Sobre esta cuestión, este crítico de arte (GREENBERG, 1948: 369) plantea lo siguiente:

Si grandes artistas como Picasso, Braque y Léger han decaído tan penosamente sólo puede deberse a que las premisas sociales generales que solían garantizar su funcionamiento han desaparecido de Europa. Y cuando uno ve, por otro lado, cuánto ha subido el nivel de la pintura norteamericana en los últimos cinco años, con la aparición de nuevos talentos tan llenos de energía y contenido como Arshile Gorky, Jackson Pollock, David Smith -entonces uno se ve obligado, para su propia sorpresa, a concluir que la premisa principal del arte occidental ha emigrado por fin a Estados Unidos, junto con el centro de gravedad de la producción industrial y el

1 Sobre esta cuestión, se presenta también muy relevador en especial el texto de SAUNDERS (2013); así como los trabajos más teóricos de Nono en los años 50 y 60, donde se reflexiona en torno a la relación musical entre Norteamérica y Europa, a la vez que analizaba los componentes sociales y revolucionarios de diversos compositores. 
poder político-.

El salto a la abstracción norteamericana, después de la Segunda Guerra Mundial, coincidió con el comienzo de numerosos compositores norteamericanos, que empezaron a tomar un relevo significativo ante sus colegas europeos, siendo algunos de estos nombres Milton Babbitt, quien contactaría con Arnold Schoenberg, después de abandonar la Alemania nazi. De hecho, Babbitt se convertiría en uno de los principales expertos americanos en la técnica de los 12 tonos de Schoenberg. Desde finales de los años 40, se generan posturas eclécticas y profundamente experimentales, destacando sobre todo en esta línea Morton Feldman, Christian Wolff y Earle Brown, en definitiva, propuestas que se encarrilarían, tal y como lo plantea Ulrich Dibelius, hacia una «des-subjetivación de la música» (YÁÑ̃E, 2006: s. p.), así como la búsqueda de las distintas oportunidades que se brindaban con las monotonías formales del minimalismo en la música.

La influencia que procedía del ámbito pictórico resultaba relevante, por ejemplo, a principios de los años 50, mientras Milton Babbitt estaba añadiendo ritmo a la base de los 12 tonos y Elliott Carter estaba experimentando con las estructuras tempo, Cage y Feldman estaban pasando el tiempo en el Cedar Bar de New York con los pintores Jackson Pollock, Willem DeKooning, Philip Guston, Robert Rauschenberg y Jasper Johns, entre otros.

La abstracción musical se convirtió en la nueva estética de los años 40 y 50, una época donde se encontraron compositores de la abstracción atonal, que algunos casos eran difícilmente comercializables, planteándose que el oyente no asumiera la composición musical como una simple mercancía de uso generalizado y altamente comercializable. Para algunos pensadores como Paco YÁñez (2006: s. p.):

La relación del compositor con los intérpretes, a través de la partitura y de la apertura de las obras, es uno de los ejemplos que Rich cita recurrentemente para distanciar en aspectos conceptuales profundos y significativos buena parte de la obra de Cage y Feldman, por citar un elemento constitutivo característico de la composición musical. Esto mismo lo podemos aplicar al Expresionismo Abstracto pictórico, una de las manifestaciones plásticas, en mi opinión, más bellas y trascendentes de la historia del arte, pero en la cual la convivencia de estéticas, estilos y principios creativos hace complejo hablar, de nuevo, de escuela en sentido estricto.

En esta contextualización norteamericana, y a pesar de algunas voces escépticas, uno de los referentes surgidos de esta inyección económica sería la denominada New York School, tanto en lo que se refiere al contexto musical, como a la faceta más pictórica, siendo el espacio germinal para el nacimiento del Expresionismo Abstracto. De igual manera, esta ebullición artística también se extendería a la danza con la Merce Cunningham Dance Company. Para Eric SMIGEL (2003: 249):

La Escuela de Nueva York se refiere primeramente a los artistas expresionistas abstractos de los años 40 y 50 , pero pronto el término fue aplicado a un grupo contemporáneo de músicos que habían desarrollado relaciones sociales y profesionales con los pintores. Los compositores Earle Brown, John Cage, Morton Feldman y Christian Wolff, junto al pianista David Tudor, fueron un grupo muy unido: se solían reunir regularmente para compartir ideas y darse apoyo unos a otros, producían los conciertos musicales de los otros, e incluso por un tiempo estuvieron viviendo cerca unos de otros. 
Este grupo de compositores musicales, que serían englobados bajo la etiqueta de New York School, impulsaron un camino decidido hacia la abstracción musical en paralelo y acompañado por los trabajos de los pioneros del Expresionismo Abstracto (Fig. 1). Todo el ambiente era propicio para el desarrollo de la experimentación abstracta, lo que venía impulsado, según Giulio Carlo ArGAN (1948: 331) «no ya en relación con sus objetos o contenidos, sino en sí mismo, en el acto de constituirse, el arte abstracto tiende a rebasar la fenomenología del hecho artístico en su propio desarrollo y su transformación, por ende, en pura experimentación». Todo este ambiente orientado hacia las nuevas especulaciones informales en la cultura será descrito por Serge GuILbAut (1990: 128) bajo esta trama cultural:

El mismo público que leía artículos sobre la importancia del arte moderno y abstracto en revistas como Fortune, la misma gente a la que se informaba de los intentos del nuevo arte de representar lo irrepresentable e ilustrar lo impensable y que estaba por tanto dispuestas a aceptar lo impensable en su vida diaria, estaba también preparada para aceptar los goteos de Pollock sin demasiado asombro, sobre todo dado que en estos momentos (finales de 1946) la obra de Pollock estaba bastante próxima a la representación de la fragmentación y la desintegración.

En las décadas de los años 40 y 50, Nueva York fue un crisol de artistas, poetas, escritores, coreógrafos y músicos. Según Eric SMigel (2003: 250), «Cage fue el primero de los músicos de Nueva York en hacerse amigo de los pintores, dio conferencias en The Club, el primer lugar de encuentro de los expresionistas abstractos, y colaboró con el artista Robert Motherwell y el crítico de arte Harold Rosenberg en cuestiones concretas dentro del periódico Possibilities (1947)».

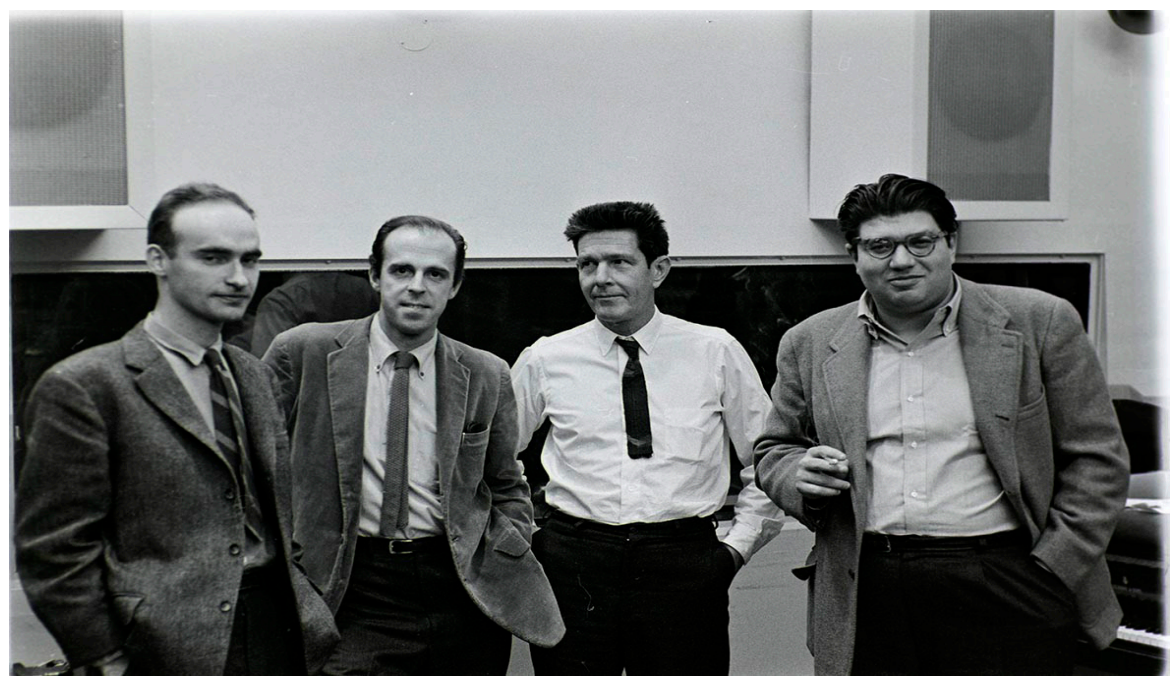

Figura 1. Fotografía conjunta de Christian Wolff, Earle Brown, John Cage y Morton Feldman (1962). Fuente: The Paul Sacher Foundation. 
Mientras la abstracción dominada la mayoría de los círculos artísticos, esta tendencia se veía complementada por los anteriores compositores, que comienzan a trabajar en los lindes de la pura experimentación, siendo remarcable el caso de John Cage, quien se introduciría en el sonido de todo aquello que se acerca a la complejidad abstracta, simulando una prescripción y musicalización del auténtico caos de la vida real, consiguiendo relanzar los verdaderos sonidos y no las estructuras complicadas de fondo. Por otra parte, John Cage había tenido una relación de amistad con Robert Rauschenberg, extrayendo de sus prácticas artísticas aspectos que posteriormente serían amoldados al interés del músico. No obstante, su experimentación antecedió los intereses materializados del mismo Rauschenberg, cuando ejecuta su trabajo 4'33", presentado en 1952 y consistente en cuatro minutos y medio de silencio, junto con sonidos medio ambientales. Anteriormente, Cage había investigado con una serie de combinaciones aleatorias, que generaba mediante el empleo de un tocadiscos o radio a modo de instrumentos musicales en Credo in US (1942). Igualmente, sugería al operador que utilizara clásicos como Dvorak, Beethoven, Sibelius o Tchaikovsky, para alterar e intercambiar las piezas como si fueran un collage para piano y percusión.

Si el Expresionismo Abstracto comenzó a perder protagonismo ante la aparición del arte pop y las diferentes tendencias experimentales de los años 60, en cambio, para Kyle Gann, los compositores de la abstracción moderna llegarían a dominar la escena musical hasta los años ochenta en pleno auge de la posmodernidad. Aunque se debe anotar que los años 70 fueron años de confluencia con la música derivada del minimalismo y la libertad de la improvisación, la música pop y de carácter más étnico, así como la aparición de los DJs. No obstante, estas colaboraciones pintura-música se seguirían dando de manera constante y paulatina en el tiempo, sirva por citar un ejemplo, las aportaciones técnicas del sobrepintado monocromático Übermalungen de los años 60 en base al trabajo colaborativo de Arnulf Rainer y Wolfgang Rihm. En cierta manera y como comenta Román DE LA CALlE (2007: 97):

¿Hasta qué extremo, pues, las relaciones y confluencias entre música y artes plásticas, cuando de hecho se presentan drásticamente, implican esa atracción por lo prohibido (Klee), suponen el derrumbe del murus aheneus para fomentar una total interpenetración (Boulez) y auspician un cierto anarquismo, siempre en favor de la multiplicidad, la vocación diaspórica y la descentralización (John Cage)?

\section{LOS COMIENZOS INTERDISCIPLINARIOS DE EARLE BROWN}

A pesar de que Earle Brown (1926-2002) es principalmente conocido como compositor musical, su relación con la pintura y la literatura, facetas practicadas en los años en que estuvo viviendo en Denver, han resultado relevantes para entender su desarrollo profesional. Este carácter poliédrico enfocado en la cultura contemporánea demuestra una amplitud de miras con su entorno y la necesidad de entretejer conexiones interdisciplinares, como mecanismo de enriquecimiento creativo. Por ejemplo, personalidades culturales como Kenneth Patchen, en el ámbito poético-literario, influyeron al compositor mediante una valiente estructura gráfica, que es desarrollada a finales de los años 40, lo que generó un estilo divergente de la partitura, tal y como se puede observar en December (1952), 
siendo planteado como una implicación experimental en el apartado gráfico, donde también se combinan la intercesión de otras disciplinas, como la estética del movimiento surgida desde los trabajos de Alexander Calder, que ya habían sido observados por Brown en 1948-49. De hecho, en el libro de Michael NyMAN (1999: 56) se describe este préstamo cultural desde la siguiente perspectiva: «la función creativa del no control y aspectos encontrados del trabajo dentro del proceso de hacer el propio trabajo, las integrales pero impredecibles variaciones flotantes de un móvil de Calder...».

Las posibilidades de generar notaciones y estructuras de bloques intercambiables en algunas de las piezas de Brown le acercaban a los móviles de Calder, ya que el músico tenía la posibilidad de una mayor decisión, a la vez que se mantenía un cierto control sobre la obra, pero siempre bajo las dinámicas de la apertura y cambio. Posteriormente, este tipo de prácticas serían apreciadas como Open Form, tal y como se puede comprobar en Twentyfive Pages [for 1-25 Pianos] (1953) y Available Forms I (1961), donde el músico puede interpretar la partitura de diferentes maneras, tal y como un espectador puede interactuar con un móvil cinético. Todo ello marcado por una construcción de numerosas posibilidades, al igual que se estipula en el arte cinético, cuando asume pautas de mayor liberalización interactiva. Asimismo, destaca Calder Piece (1966), una pieza elaborada para cuatro percusionistas, donde el propio escultor diseña un móvil específico para la composición musical, que adquiriría un carácter directriz para las cuatro baterías de percusión, dictando diferentes combinaciones y secuencias de acuerdo con los distintos movimientos adquiridos.

Esta propensión hacia lo aleatorio era algo propio no sólo de ciertas piezas del arte cinético, sino especialmente del ámbito del Expresionismo Abstracto, donde las premisas de la producción bajo la seña de identidad del azar y la indeterminación finalmente determinan el resultado final, que sería descrito por el crítico de arte Achille Bonito Oliva (2002: 44) de este modo:

Aquí hay una escritura que se repite pero que afirma su derecho. Más bien la omnipotencia del gesto irrepetible e individual que proviene de la energía del lenguaje. Pero la irrepetibilidad, para ser tal, requiere método y disciplina, la adoptada por el artista americano a través de la asunción de la constante de estructura del círculo.

Anteriormente, las aportaciones del dodecafonismo permitieron a este compositor abordar nuevas posibilidades para el ritmo y su variación, fomentando variantes dinámicas y rítmicas, que quedarían reflejadas en su trabajo Three Pieces for piano (1951). Otra referencia palpable sería su interés por los valores estocásticos, que planteaba Xenakis, mediante combinación de valores matemáticos gráficos, que provenían de Schillinger.

Earle Brown se especializó en el sistema serial de composición musical de Webern, así como en el análisis según Schillinger, ya que observó la existencia de un cierto agotamiento en los medios de la notación tradicional, lo que no le permitía toda la dilatación expresiva que necesitaba. De este modo, sus comienzos musicales remiten a un entramado compositivo, que se acercaba a los postulados de carácter más científico y matemático de Schillinger, así como a las bases serialistas en torno a Karlheinz Stockhausen. También, su metodología compositiva se aproximaba a las disposiciones de Messiaen o Boulez, que son permutadas en series de variación que se proyectan constantemente. 
Estas serían algunas de sus primeras y principales influencias de formación, ${ }^{2}$ que curiosamente en el apartado musical se acercan más al ámbito europeo que a la figura de John Cage, de ahí que la posición de Earle Brown se haya asumido como un nexo conectivo entre la cultura de posguerra entre Estados Unidos y Europa. No obstante, para David Revill (1992: 139-140), Brown compartía con John Cage numerosos intereses comunes dentro del apartado de la filosofía, el arte y obviamente la música. Después de conocer a Cage y Cunningham en 1952, se traslada con su mujer a Nueva York, donde empieza a plantearse un trabajo en relación con la música electrónica, colaborando con el propio Cage, Tudor y el matrimonio Louis y Bebe Barron en sus experimentos electroacústicos, siendo resultado de este proceso experimental en 1952-55 propuestas como Octect I for Eight Loudspeakers (1953), que es relacionada con el Poème électronique (1957-58) de Edgar Varèse, junto a otras propuestas de carácter más serialista por parte de Brown en Perspectives (1952) o Music for Violin, Cello and Piano (1952). Son años donde trabaja y aprende de los trabajos de John Cage and David Tudor, especialmente cuando toma parte en Project for Music for Magnetic Tape (Holmes, 2005: 108). De acuerdo con D.J. HoEK (2004: 355):

A pesar de que Brown trató de proporcionar a los oyentes una variada muestra de música de vanguardia, admite que su intención no era del todo objetiva. Por el contrario, con diverso repertorio de las series, Brown también intentó establecer un contexto internacional dentro del cual promover el trabajo de los compositores experimentales americanos.

Recordemos que David Tudor ejecuta Perspectives y Three Pieces for Piano en el Black Mountain College el 27 de junio de 1953. Dicha escuela de arte permitió a Brown familiarizarse no sólo con los fundamentos teóricos de la abstracción geométrica, siendo uno de sus principales difusores y docentes Joseph Albers, sino también conocer la obra de Richard Buckminster Fuller, quien en 1949 diseñaría su primera gran cúpula geodésica. Este compositor descubre el artículo redactado por Elaine de Kooning en 1952 en Art News Magazine sobre la obra de este arquitecto, donde aparece el término de "sinergia», junto con la definición aportada por Fuller, siendo explicado de este modo por BROWN (1989: s. p.):

Sinergia es la energía liberada por dos energías que se cruzan, y la energía resultante de las dos energías que se cruzan no se puede predecir exactamente en relación con la causa de cualquiera de las energías originales. En otras palabras, la energía está más allá, es más que la suma de sus partes. Pensando en esto y leyendo ese artículo y la definición de sinergia de Bucky, se me ocurrió que lo que estoy tratando de hacer con estas partituras gráficas, November 1952, subtitulado Synergy, es ponerlo allí. (...) El número de diciembre sale en noviembre, y obviamente lo leí y puse esa sinergia en November 1952. Lo que pensé fue: tengo un concepto para escribir una pieza, luego lo pongo en papel, así mi concepto es una energía y lo que pongo en el papel es otra energía. Se lo doy a David Tudor, por ejemplo, y esa es una tercera energía. Cuando estaba haciendo estas tareas gráficas, el resultado se podía ver que no residía únicamente en mi concepto o en mi hoja de papel o en mi acción, o en la de David, ya que había permitido suficiente flexibilidad y espacio en el proceso para que el resultado fuera impredecible...

2 Para profundizar sobre los contenidos teóricos y musicales de este autor, se puede consultar KIM, R.Y. (2017). Otras opciones de interés serían los propios textos redactados por el compositor, así como las entrevistas recibidas, que se pueden encontrar en el siguiente enlace: http://archivetest.earlebrown.org/search/1/all/2-Writings-of-Earle-Brown [Consulta: 21/12/2021]. 


\section{DE LOS DRIP-PAINTINGS A LA FORMA ABIERTA DE EARLE BROWN}

Jackson Pollock (1912-1956) también había tenido un acercamiento al ámbito musical, aunque fuera de una manera más somera, de hecho, uno de los episodios más conocidos fue en 1951 cuando el fotógrafo Hans Namuth realiza un documental sobre la técnica pictórica del artista, especialmente con la intención de documentar el proceso de elaboración del dripping y la aplicación del goteo y el resto de los elementos pictóricos de manera aleatoria. El trabajo documental que realizó Hans Namuth se llevó a cabo durante todos los fines de semana de octubre y noviembre. Este primero le propuso pintar sobre una superficie de cristal debajo de la cual, se colocaría la cámara. Para ello, el pintor realizó un trabajo sobre un soporte de vidrio, de tal manera que fue filmado desde la parte de abajo, siendo titulada N. 29, de este modo, el espectador podía ver con absoluta claridad no sólo el proceso pictórico, sino la actitud y el rostro de un Pollock, trabajando bajo los condicionantes del inconsciente. A la hora de montar la película, el productor del documental Paul Falkenberg quiso introducir una música exótica, procedente de la cultura indonesia. Ante el rechazo de los Pollock por articular un tipo de música que no empatizaba con la manera de trabajar de Jackson, su mujer Lee Krashner conectó con Morton Feldman para estudiar la posibilidad de incorporar su música al documental.

Por otro lado, Pollock había recogido algunos de sus títulos de varias canciones, tal y como ocurre con Full Fathom Five (1947). El título, que fue tomado de la canción de Ariel en la obra La tempestad de William Shakespeare, se debe a Ralph Manheim, vecino de Long Island, que se había dedicado a traducir obras de Carl Gustav Jung. Este mismo personaje también había elegido el título de Alchemy (1947). De hecho, Pollock le estaba muy agradecido a Ralph Manheim por la comparativa que había trazado entre la obra de Jung y su obra pictórica.

Debemos recordar que Harold Rosenberg había acuñado el término de Action Painting, para definir el trabajo activo de pintores como Jackson Pollock, Willen de Kooning y Franz Kline. En su artículo seminal titulado The American Action Painters (1952), comenta lo siguiente sobre dicha denominación:

En un cierto momento el lienzo comienza a aparecer después de otro a un pintor americano como si fuera una arena en la cual actuar -más que un espacio en el cual reproducir, rediseñar, analizar, o expresar un objeto, actual o imaginado. Lo que había que desarrollar sobre el lienzo no era una imagen sino un evento (ROSENBERG, 1952: 22).

En este sentido, el concepto de evento y acto era una manera de encuentro del artista con el material de trabajo. Si la pintura para un expresionista abstracto era un acto, de igual manera debería entenderse la actitud de los músicos asociados con la Escuela de Nueva York ante la composición, donde el evento era el encuentro entre el músico y la partitura. El sentido de la liberación artística, que se promovía entre los pintores y músicos vinculados a dicha escena cultural remitía tanto a la ejecución pictórica como a la hora de componer las partituras, generando una sensación de mayor libertad, no sólo en el propio artista o compositor, sino en el oyente y el espectador, y por ende en la misma obra. Earle Brown realiza el siguiente comentario sobre su relación con diversos artistas:

Feldman y yo solíamos salir con Bill de Kooning y con Rothko. Rothko no estaba 
mucho por allí, pero sí Bill de Kooning, Pollock y esas personas. Así crecimos en una especie de transformación artística. ¡Fue muy emocionante! Los expresionistas abstractos de Nueva York en los años cuarenta y cincuenta fueron un poderoso movimiento de arte americano (DuffIE, 1991: s. p.).

Estos trabajos que venían caracterizados por la multiplicidad de ejecuciones diversas, distorsionando el propio material sonoro en su contenido, generando una tensión entre interpretación-composición, sería algo atribuible igualmente a la espontaneidad ejecutiva e interpretativa de la Action-Painting, entendido como energía inductiva que facilita los cauces liberadores de la propensión creativa, de hecho, para Achille Bonito Oliva (2002: 27): «Como antes la vanguardia histórica, también el Action Painting inviste al arte la esperanza de una posible transformación del mundo de una palingenesia total que debe liberar al hombre de toda alienación, que liberara al individuo y la historia».

Tal y como habíamos comentado previamente, el interés intelectual de Brown no sólo se había centrado en la pintura, sino que también había abordado cuestiones poéticas desde los escritos de James Joyce y la poesía de Gertrude Stein y Kenneth Patchen. Esta influencia recibida desde la comunidad vanguardista ha sido tanto desde el ámbito filosófico como práctico. No obstante, junto a la composición gráfica de Patchen, deberíamos subrayar la movilidad de las propuestas de Alexander Calder y la espontaneidad ejecutiva de Jackson Pollock. Brown había descrito a este pionero de la abstracción pictórica como una de las primeras influencias predominantes de sus trabajos mediante «la direccionalidad y la espontaneidad de los resultados de Pollock en relación con los materiales y su particular imagen del trabajo...como un espacio total (de tiempo)» (BROWN, en Cox y Warner, 2004: 189). De ahí que resulte admisible la sugerencia comparativa del trabajo de Brown en Available Forms I (1952) con la propia espontaneidad generada por Pollock. Algunas características de esta composición conllevaban «el repunte puntillista de la partitura mediante instrumentos individuales, acordes de diferentes densidades y duración, goteos de arpas brillantes y percusión, y gestos que van desde lo tenue a lo audaz, espejo de lo que se puede ver en un lienzo de Pollock» (KozINN, 2007: s. p.). (Fig. 2).

Las obras de este expresionista abstracto resultaban profundamente originales en sentimiento, pero fuertemente ambiguas en forma. Durante los años 1944 y 1945 las pinturas continuaron explorando las posibilidades de la totalidad sin realizar un completo compromiso con el goteo. De hecho, sus trazos en algunos momentos se volvieron más caligráficos, caso de Night Mist (1945) y con carremolinamientos de escritura a mano automática. Es en estos años cuando empieza a distribuir formas dentro del lienzo al igual que lo había hecho en sus dibujos "psicoanáliticos», tal y como se puede comprobar en propuestas como Gothic y Night Ceremony (ambas de mediados de los años 40), donde cada forma individual existe independientemente en su propio intervalo espacial. 


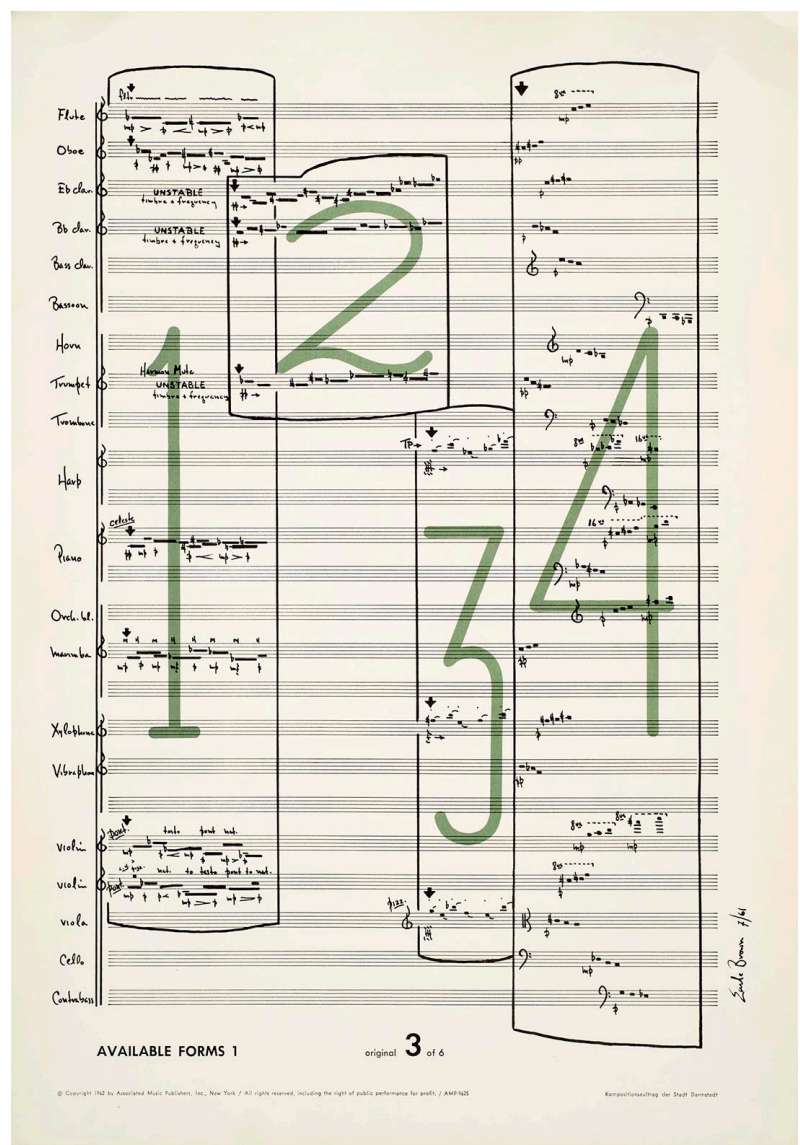

Figura 2. Earle Brown. Available Forms I (1952). Fuente: The Paul Sacher Foundation.

El lenguaje personalizado de Brown iba adquiriendo cada vez más singularidad, ya que a las variables habituales del sonido (timbre, duración, intensidad y altura), ahora le incorpora otra nueva variante: la del intérprete, siendo una condición de variación en la pieza y también como elemento de cierre de la serie, lo que permitía al músico disponer de un margen muy consistente en el momento interpretativo de la partitura. Esto significaba que el compositor no dejaba la pieza cerrada y que el músico tenía opciones de continuar marcando variables. Brown manifestó lo siguiente:

Nunca he estado avergonzado por escribir una bella melodía, un pasaje muy lírico, o lo que yo considero una bella progresión de acordes. A la vez también he estado interesado en activar la interacción entre compositores e intérpretes, y en hacer una música de una mayor colaboración mundial, no en todos los casos, pero sí en algunos (Pytheas Center For Contemporary Music, s. f.: s. p.).

Sus técnicas compositivas y experimentales con las notaciones temporales, la improvisación y la estructura compositiva de forma abierta se han convertido 
en una metodología para la composición contemporánea. Por otro lado, la procedencia conceptual de Earle Brown a la hora de generar piezas no finalizadas, sobre la forma y con infinitas combinaciones, mantenía una directa conexión con los planteamientos de Jackson Pollock cuando lanzaba su pintura al lienzo, produciendo composiciones caracterizadas tanto por líneas como por manchas cromáticas yuxtapuestas con resultados aleatorios y marcados por el azar en muchos casos. Esta actitud derivada de las prácticas automáticas también fue impresa en los trabajos de Robert Rauschenberg, que conjuntaba y yuxtaponía imágenes y escenas de collage sin ningún nexo de lógica aplicativa, al igual que se puede observar en sus combine-paintings y objetos conexionados de diferentes procedencias. De idéntico modo, este juego de yuxtaposiciones se mantendrá en su repertorio compositivo musical, sirva de ejemplo una de sus últimas propuestas: Summer Suite'95 (1995), donde las constantes de la composición, interpretación y anotación se yuxtaponían y simultaneaban en el momento que Earle Brown interpretaba dicha pieza al piano, mientras un ordenador la transcribía a modelos gráficos.

La metodología pollockiana de unir diferentes realidades y episodios del inconsciente en un mismo plano de representación sería tenido en cuenta por Earle Brown, tal y como se puede apreciar en el siguiente comentario, donde parafrasea a Gerard de Nerval: «El surrealismo es la yuxtaposición de realidades diferentes entre sí en el mismo plano de la realidad (Gerard de Nerval). El ejemplo fue un paraguas y una máquina de coser haciendo el amor sobre una mesa de disección. Los surrealistas y los dadaístas eran realmente unos rompedores a la hora de impactar en la complacencia de la gente» (DufFIE, 1991: s. p.). En conexión con esta dinámica, Jeanne SIEGEL (1999: 204) anota este otro planteamiento:

Ha sido atribuido un gran papel a la influencia de las ideas jungianas en el Expresionismo Abstracto, particularmente en Pollock, debido a la temprana influencia de John Graham y su propia terapia. Pollock ha confirmado su creencia en el poder del inconsciente y en la presencia del mito en la obra, lo que le ató a las ideas del inconsciente colectivo. Sin embargo, los comentarios de Pollock fueron también dirigidos a concluir que él había absorbido ideas a través de la lectura concentrada.

Jackson Pollock había coincidido varias veces con Brown en Nueva York, introduciéndole la posibilidad de realizar múltiples lecturas sobre la abstracción pictórica, de ahí la atracción que mostraba Brown por la aleatoriedad de sus formas. Esta manera de trabajar del pintor se sustentaba, como ya hemos comentado, en el automatismo psíquico, tomando

del surrealismo el interés por la acción de pintar, por el proceso, más que por la obra acabada, rompiendo así una tradición de siglos. Gorky decía que nunca terminaba un cuadro y le gustaba pintar porque era algo que no tenía fin. Pollock, que toma como un cumplido el comentario despectivo de un periodista en ese mismo sentido va a hacer precisamente de esa acción de pintar la razón de ser de su obra. Sólo faltará que alguien le aplique el calificativo y eso lo hará Rosemberg en 1952: Pintores de acción americanos. El proceso creativo es lo importante, es un «momento» en la vida del artista y ese proceso es el que filmó Hans Namuth, como un primer happening, en octubre de 1950, mientras Pollock fabricaba dos obras, una de ellas sobre vidrio (GARCíA, 1996: 220).

En el caso de Earle Brown, serían los intérpretes los responsables no sólo de llenar de contenido, sino igualmente de dar forma a los elementos planteados por 
el compositor. En este sentido, la escritura se convierte en una práctica unívoca que al ser interpretada asume un cuerpo estético independiente. La partitura ya no es en sí misma una obra finalizada, sino que admite la posibilidad de producir nuevas interpretaciones.

La apuesta por la libertad del intérprete en los trabajos de Earle Brown recuerda la libertad de ejecución de los expresionistas abstractos y por ende el modelo interpretativo del propio espectador al observar la obra pictórica, pero asumiendo una serie de mínimos en base al automatismo psíquico y su exploración en el ámbito de las emociones del pintor. De hecho, esta libertad de implicación hacia el intérprete también venía marcada por una serie de condicionantes estipulados en la partitura, como punto focal de partida. Brown parafrasea a Gertrude Stein en esta sentencia:

La vida no se basa en un principio, un desarrollo y un final (Gertrude Stein). Nunca sabes cuándo es el medio y cuándo es el fin. Eso me ha influido en relación con el concepto del tiempo. En mis primeras anotaciones, he escrito que la próxima cosa interesante que suceda en la música será una revisión de la naturaleza de la continuidad, la retórica y el tiempo (DiCKINSON, 1987: s. p.).

En este mismo sentido, recordemos las siguientes palabras de Pollock: "Cuando pinto tengo siempre una idea general de lo que estoy haciendo. Puedo controlar el fluir de la pintura; no hay nada accidental, de idéntica manera que no existe ni principio ni final» (JACHEC, 2011: 128). Resulta clarificador el comentario de Elizabeth FrAnK (1983: 68) al respecto, al afirmar que Pollock «dijo que no usaba el accidente, que, de hecho, negó el accidente. Pero lo negó por tratar de buscarlo, al asignarlo un papel marginal pero coherente». Dentro del entramado de la misma pintura derramada, el artista inyectó un estilo, bajo una potencia polimórfica, con la capacidad para estar en todas partes a la vez, y servir el final entre la ilusión y la materialidad, desarrollando pinturas con unas estructuras tanto internas como inmediatas, tal y como podemos observar en Alchemy (1947) y White Cockatoo: Number 10 A (1948), donde crea figuras de áreas que se superponen, así como entramados biomórficos, que implican capas enterradas de figuración que componen la abstracción de su pintura.

Este representante de la abstracción norteamericana y su técnica del dripping habían generado una fuente de inspiración para Earle Brown, tal y como afirma Owen MEYers (2001: 3), «resulta de mucha influencia las artes visuales en la música de Brown, proviniendo de Piet Mondrian, Jackson Pollock y Calder. La influencia de Mondrian tiende a la naturaleza gráfica de las notaciones de Brown, Pollock añade espontaneidad y Calder proporciona movilidad». Ciertamente, una de las piedras angulares no sólo para Earle Brown, sino para otros muchos intelectuales y creadores, había sido Jackson Pollock, de hecho, tal y como comenta Serge Guilbaut (1990: 246): «En 1949 Pollock se convirtió en el esperado héroe cultural con la suficiente estatura para formar a su alrededor algo parecido a una escuela. Él fue el catalizador, el rompehielos de la nueva vanguardia norteamericana».

La aportación disciplinar de Brown se concentraba principalmente en los componentes estructurales de las piezas sonoras, lo que vaticinaba una cierta correlación con la manera de trabajar de los expresionistas abstractos y de manera especial con las drip-paintings. Al respecto, Paco YÁÑEz (2006: s. p.) desarrolla la posterior reflexión: 
Se me hace imposible no pensar en una suerte de dripping sonoro (término que hace poco utilizaba para referirme a los Exercises (1973-75) de Christian Wolff) cuando escucho piezas como Times Five (1963), de centelleante serialismo percutivo, casi como las gotas del pincel de Pollock al golpear el yaciente lienzo, del cual siempre alguna línea se derivaba hacia los márgenes, como queriendo prolongar su búsqueda y agonía hacia los límites del vacío insondable emplazados a ambos lados de la tela; algo que en la música de Brown también parecemos escuchar cuando prolonga alguna de estas notas golpeadas contra el intérprete, que en un primer momento busca una salida cuyo final será, en todo caso, un conjunto definido, como vemos, en mayor medida, en la música del Brown de los sesenta.

En el trabajo documental de Hans Namuth, Pollock muestra una serie de movimientos que no llegan a instalarse dentro del trance, pero que tampoco son rutinarios. En cualquier caso, hay dos aspectos que dominan el proceso, como son la espontaneidad y la impronta de lo directo para ver el proceso creativo del pintor, teniendo en cuenta que en este movimiento pictórico se daba un consistente protagonismo al proceso ejecutivo más que al resultado final. Este trabajo audiovisual lo convirtió en un héroe de la espontaneidad inarticulada, un hombre que pintaba en cierta manera su propia biografía, que rompían las barreras entre el arte y la vida. En este sentido, el acto de pintar se había convertido en una especie de «ritual religioso que le ayudaba a mantenerse en un continuo estado de delirium lúcido» (TOMASSONI, 1978: 5).

A Brown le interesa las múltiples lecturas posibles de una obra abstracta, lo que suponía aceptar los parámetros del no control, así como la improvisación y los aspectos del hallazgo de la obra dentro del proceso creativo, lo que en cierta manera, era una dinámica habitual del arte procesual que estipulaba Jackson Pollock. A la vez, Brown extraía sus técnicas de la manera de trabajar de Pollock, observando que a pesar de que se planteaba una aleatoriedad en el resultado final, el artista también era capaz de controlar y decidir numerosas cuestiones de la acción artística. Por este motivo, Earle Brown profesaba a su manera «unas condiciones establecidas únicas y personales de control de la totalidad» (FAcCiO, 2014: 384-385). No obstante, se cultivaba una liberación del tiempo en lo que sería una partitura que podía ser leída en cualquier posición y dirección, siendo su duración indeterminada y los símbolos entendidos e interpretados de acuerdo con los parámetros mentales del intérprete, lo que le convertía a su vez en una parte integrante de la obra. Todo esto debía ser entendido, como afirma Juan José FACCiO (2014: 384-385): «en el sentido más básico, de un conjunto de figuras abstractas que son traducidas como instrucciones e interpretadas como acciones, pero cuyo resultado es impredecible».

Incluso el juego combinatorio de dos intérpretes a la hora de llevar a cabo la puesta musical de la pieza suponía un punto de inflexión tanto en el ámbito musical, como en el pictórico. De hecho, en el apartado musical, el material podía ser dividido entre los dos intérpretes, generándose procesos de mayor complejidad en cuestiones de independencia e interacción. En cualquier caso, anotando las instrucciones del compositor, cada director decide lo que se interpreta y cuando, dependiendo lo que haga el otro. En un buen número de composiciones de Brown, la música está compuesta como módulos fijos (aunque a menudo con mezclas idiosincráticas de notación), no obstante, el orden se deja libre para ser elegido durante la actuación del director.

En definitiva, se trata de romper estructuras estáticas, alternando entre las cerradas y abiertas, lo que vaticinaba una deriva hacia postulados marcados por la 
propia ambigüedad creativa y compositiva, aspectos que claramente se remetían al Expresionismo Abstracto como al arte cinético, siendo corroboradas estas ideas por el propio Earle Brown: «Como he comentado habitualmente, los trabajos gráficos, móviles y de forma abierta que he escrito desde 1952 se inspiraron originalmente en la obra artística de Alexander Calder y Jackson Pollock» (The Earle Brown Music Foundation, 2013: s. p).

Los planteamientos de Brown sobre la obra abierta se circunscriben en un momento en que se dispara un notable interés por este tema, tal y como posteriormente lo demostrará el propio Umberto Eco con su trabajo literario Obra Abierta (1962), donde este pensador italiano analiza las distintas posibilidades de apertura del lenguaje musical, cuando se invita al intérprete o al músico a la hora de decidir sobre la composición o cerrar la estructura, para ello en su texto se recogen ejemplos compositivos de Stockhausen, Boulez y Berio. La aportación internacional de este estudio consolidaba si cabe aún más la apuesta experimental llevada a cabo por el propio Brown, lo que a la vez también reforzaba los lazos del compositor norteamericano con la propia vanguardia musical europea, mediante su estudio sobre los principios del serialismo y la exploración de la forma abierta, etc.

Si Brown planteaba la existencia de una crisis en las obras cerradas y por ende la posibilidad de generar propuestas más abiertas, donde los propios intérpretes asuman una mayor presencia direccional y procesual, ya anteriormente Jackson Pollock anunciaba que la pintura de caballete también estaba en crisis por su tendencia cerrada, haciéndolo saber en un comentario a raíz de la solicitud de una beca Guggenheim en 1947:

Me gustaría hacer grandes pinturas a medio camino entre la pintura de caballete y la pintura mural. Creo que la pintura de caballete es un género que se muere y que la sensibilidad moderna va hacia las paredes pintadas o los murales. No creo que sea tiempo todavía para llevar a cabo una transición completa entre la pintura de caballete y la pintura mural. Las pinturas que proyecto estarían a medio camino. Intentarían marcar un camino hacia el futuro, pero sin llegar del todo (Pollock, en GARCíA, 1996: 225).

El objetivo del artista no sólo era cambiar y alterar el formato habitual para aplicar el proceso pictórico, sino acercar e introducir fenoménicamente al espectador en el propio cuadro, haciéndolo habitar e insertarse dentro de los propios límites de sus dimensiones físicas, de este modo, el espectador formaría parte del mismo cuadro. De igual manera, Earle Brown abre la obra, permitiendo que el intérprete participe y genere nuevas posibilidades en el proceso de desarrollo musical. Brown formaliza las notas y la dinámica en su notación temporal, pero no quiere definir de manera conclusiva la duración, quedando una forma abierta, que rememora no sólo la obra en constante movimiento y transformación de los móviles calderianos, sino especialmente la sensación pictórica de los expresionistas abstractos al moverse en un abanico de constante dilatación temporal, como si los arabescos lineales y gráficos de Pollock no parecieran acabarse nunca, lo que generaba una sensación ad infinitum.

El interés de Pollock, con su asentamiento metodológico en el surrealismo, se centraba en ponerse delante del lienzo sin ideas previas necesariamente, y que simplemente las ideas creativas comenzaran a surgir. No se trataba de buscar un resultado previo, la obra quedaba abierta y con diferentes posibilidades tanto 
procesuales como creativas, de este modo, se asumía una búsqueda deseada de lo desconocido. Si como bien comenta Elizabeth FRANK (1983: 105) sobre el pintor: «Él creo una nueva escala, una nueva definición de superficie y toque, una nueva sintaxis de relaciones entre espacio, pigmento, borde y dibujo, desplazando las jerarquías con una poderosa estructura autogenerada, intrincada fabulosamente y sin precedentes». Por otro lado, Earle Brown consolidaba declaratoriamente este acercamiento: «Nos sentíamos muy cerca de los pintores que admirábamos. Yo estaba fuertemente influenciado por la inmediatez y la espontaneidad de Jackson Pollock y Bill de Kooning. Quería llevar el gesto espontáneo a la música, y finalmente lo hice con las formas abiertas. Estábamos buscando una nueva forma de expresión musical» (DiCKINSON, 1987: s. p.).

En 1959, con Hodograph I, Brown diseñó el contorno y el carácter de manera abstracta en lo que llamó áreas implícitas de la pieza, también aspecto analizado por Pollock. El estilo gráfico fue más gestual y caligráfico que la abstracción geométrica de December (1952), que se compone exclusivamente de líneas horizontales y verticales que varían en anchura. Este trabajo se convirtió en una pieza hito en la historia de la notación gráfica de la música, siendo el objetivo del ejecutante interpretar la partitura visual y traducir la información gráfica a la música (Figs. 3 y 4 ).

La primera pieza en forma abierta de Brown fue Twenty-Five Pages (1953), es decir, veinticinco páginas sueltas, para lo que se pidió entre uno y veinticinco pianistas. La partitura permitía que el intérprete(s) organizara las páginas en el orden que consideraran oportunas, siendo además las páginas anotadas de forma simétrica y sin claves, de este modo la orientación superior e inferior podía ser reversible. En esta obra, también empleólo que élllamaba time notation o proportional notation, donde los ritmos eran indicados por su alargamiento longitudinal, disposición que también se puede observar en algunos de los cuadros de Pollock de desarrollo horizontal (Fig. 5). Si a los oyentes de Earle Brown se les solicitaba que no asumieran unas ideas preconcebidas en base a un ritmo constante y melodías totalmente cerradas, lo mismo ocurría cuando Pollock realizaba sus trabajos y éstos quedaban abiertos a las propias especulaciones interpretativas de la representación. De hecho, Pollock escribió en la revista Possibilities:

Cuando estoy en mi cuadro, no soy consciente de lo que hago. Únicamente tras un periodo de familiarización por así decirlo veo en qué he estado trabajando. No me asusta introducir cambios o destruir la imagen, porque todo cuadro tiene una vida propia, vida que yo intento dejar que aflore. Sólo si pierdo contacto con la pintura el resultado se convierte en un caos. De lo contrario es pura armonía, un fácil toma y daca, y el cuadro sale bien (JACHEC, 2011: 127). 
De las Drip-Paintings de Jackson Pollock a la Forma Abierta de Earle Brown

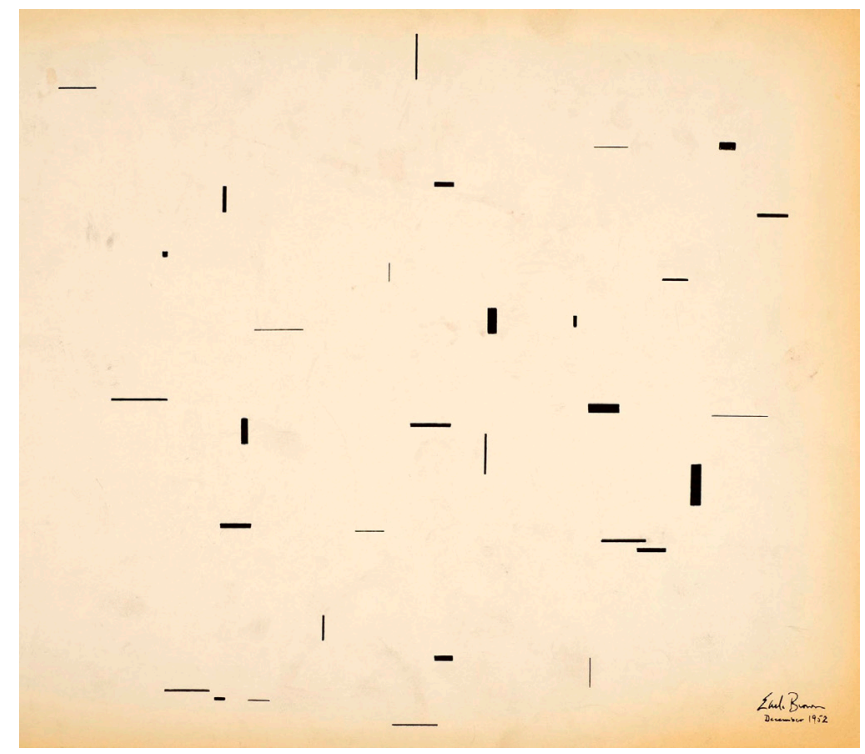

Figura 3. Earle Brown. December (1952). Fuente: The Paul Sacher Foundation.

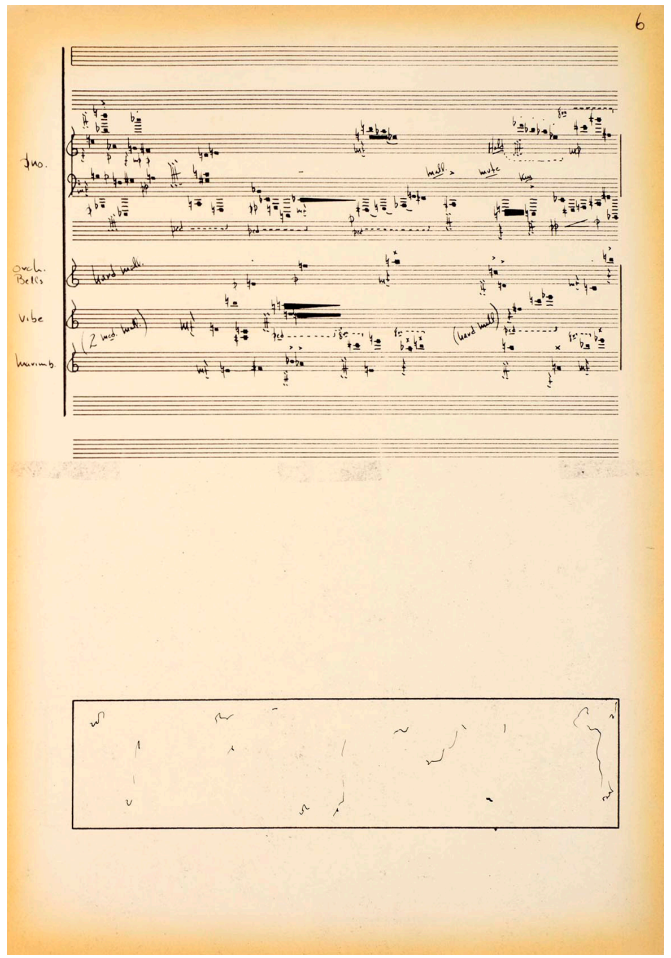

Figura 4. Earle Brown. Hodograhp I (1959). Fuente: The Paul Sacher Foundation. 


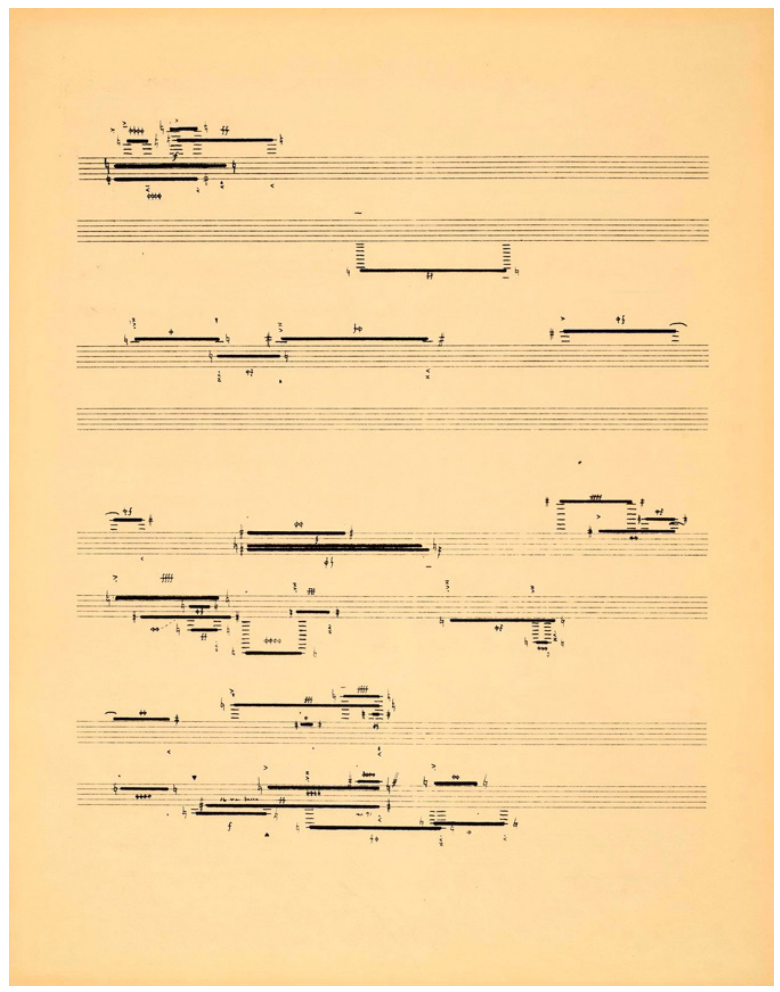

Figura 5. Earle Brown. Twenty-Five Pages (1953). Fuente: The Paul Sacher Foundation.

Las yuxtaposiciones lineales y cromáticas de Pollock eran juegos aleatorios y arbitrarios, que podían ser llevados hasta el límite de su máxima expresión. Como aclara HunTER (1958: 11-12) la pintura era «campo de batalla tras una encarnizada confrontación, cubierto en este caso por los cadáveres de Picasso, Masson y Miró y fragmentos del arte indio americano». Estas actitudes se repetirían en posteriores trabajos abordando constantemente la misma premisa de intercalar y superponer las mismas intensidades formales y cromáticas. Si esto lo lleváramos al ámbito creativo de Earle Brown, tendríamos un ejemplo cercano con el caso de los Modules I E II, compuestos en 1965-66, al igual que en su anterior Available Forms II (1962), propuesta escrita para una gran orquesta bajo la tutela de dos directores, lo que permite arbitrar dos planos simultáneos de actividad sonora. Todos los sonidos están compuestos en cada partitura, pero los dos directores tienen la posibilidad de yuxtaponer y superponer los sonidos, modificando de este modo su duración y por tanto la intensidad en una relación espontánea entre ambos durante la actuación. La propuesta plantea generar una actividad de colaboración inmediata y espontánea, que surja mediante diversas combinaciones y configuraciones formales y poéticas en cada actuación, mientras que el carácter básico de la obra se mantiene. 


\section{CONCLUSIONES}

A partir de 1948, el crítico de arte Clement Greenberg empezó a publicar diferentes artículos en revistas como Partisan Review, vaticinando no sólo la propia independencia cultural del arte norteamericano, sino incluso su posicionamiento en un pedestal de predominio hegemónico.

La aparición y evolución del Expresionismo Abstracto en la posguerra concordaría con el inicio de una serie de compositores norteamericanos, que comenzaban a desplegar unas pautas de atracción e interés creativo y experimental frente a lo que estaban proyectando sus contemporáneos europeos. En este sentido, a partir de finales de la década de los años 40, toman relevancia y protagonismo productivo nombres como Morton Feldman, Christian Wolff y Earle Brown, entre otros tantos. Esta correlación pictórica y musical en favor de una abstracción experimental marcaría el carácter estético y cultural de los años 40 y 50 en los Estados Unidos, y por ende de la Europa cultural. Esta simbiosis concomitante se transformó en el foco de la denominada New York School, convirtiéndose en el punto germinal del Expresionismo Abstracto.

La implicación poliédrica de Earle Brown (1926-2002) con la cultura circundante obliga a dirigir la mirada deductiva no sólo al campo de la composición musical, sino a atender su interés por la pintura y la literatura, aspectos que habían sido experimentados durante los años en que estuvo viviendo en Denver. Este carácter multidisciplinar y analítico en relación con la cultura contemporánea marcaba una apuesta metodológica, que urdía parentescos interdisciplinares, como mecanismo de complicidad creativa. Si el aliciente literario-poético provenía de su acercamiento a Kenneth Patchen, James Joyce y Gertrude Stein, en cambio, la aproximación al ámbito artístico se generaba a partir de sus fluidas relaciones con Robert Rauschenberg, Jasper Johns, Cy Twombly, Lichtenstein, Willem de Kooning y Mark Rothko. No obstante, su implicación creativa se vio especialmente influenciada por las técnicas pictóricas de Jackson Pollock, que vaticinaba de una manera más directa el uso de la espontaneidad, el azar y la indeterminación procesual de la obra.

Los contactos que se mantuvieron entre Jackson Pollock y Earle Brown, cuando coincidían en Nueva York, se tradujeron en una fructífera relación de intercambios reflexivos, pero sobre todo al músico le permitió ser consciente de la posibilidad creativa que ofrecía la aleatoriedad de sus formas. La aportación ejecutiva de Pollock, extrayendo pautas tácticas desde el surrealismo, se asentaba en situarse frente al lienzo sin ideas previas y que únicamente los pensamientos creativos empezaran a surgir. En ningún caso, se pretendía obtener un resultado preconcebido, quedando la obra abierta y con diferentes posibilidades tanto procesuales como creativas, de este modo, el pintor se sumergía en una indagación hacia lo desconocido. Esta libertad de actuación compositiva vigorizaría la propia apuesta de Earle Brown en favor del Open Form, favoreciendo de este modo la libertad del intérprete en el momento de la ejecución musical. Sus propuestas vendrían marcadas por una multiplicidad de ejecuciones diversas, que permitía alterar el material sonoro en su contenido, así como fomentar una mayor tensión entre interpretación-composición, y todo ello impulsado por la aceptación de la espontaneidad ejecutiva e interpretativa de la Action-Painting, siendo aplicado a modo de metodología que permita producir cauces emancipados en la práctica creativa. 
Partiendo de premisas analíticas y reflexivas en torno al modelo de trabajo de Pollock con las drip-paintings, el propio lenguaje de Brown asumía cada vez una mayor singularidad, ya que además de las variables tradicionales del serialismo (timbre, duración, intensidad y altura), ahora se le añade otra nueva variante: la del intérprete, lo que alteraba la condición de mutación en la pieza, concediendo al músico un mayor protagonismo en el proceso interpretativo de la partitura.

En cierta manera, se había conexionado la inclinación conceptual de Earle Brown a la hora de generar piezas no finalizadas, con la opción abierta sobre la forma y sus ilimitadas combinaciones, que se experimentaban en las drippaintings de Jackson Pollock. Si el proceso pictórico del pintor permitía la ejecución de una obra abierta de infinitas posibilidades, igualmente la partitura ya no sería en sí misma una obra finalizada, al asumir la probabilidad de insertar nuevas interpretaciones. Si Brown extrajo parte de sus técnicas del modus operandi de Pollock, observando las diferentes opciones de la aleatoriedad en el proceso efectuado, también era consciente en cierta manera de la necesidad de controlar y decidir numerosas cuestiones de ejecución musical. Tanto las técnicas compositivas y experimentales con las «notaciones temporales» de Earle Brown, como el uso de la improvisación y la estructura compositiva de forma abierta se han transformado en un componente esencial para entender la composición contemporánea.

\section{REFERENCIAS}

Adorno, T. (2002): Essays on Music. Selected, with Introduction, Commentary, and Notes by Richard Leppert, University of California Press, Berkeley and Los Angeles, California.

ADORno, T. (2003): Filosofía de la nueva música, Ediciones Akal, Madrid.

ARGAN, G. C. (1948): Collezione Peggy Guggenheim. Catálogo de XXIV Biennale di Venezia.

AviÑoA, X. (ed.) (2014): Tecnología y creación musical, Editorial Milenio, Lleida.

Beal, A. C. (2006): New Music, New Allies: American Experimental Music in West Germany from the Zero Hour to Reunification, University of California Press, Los Angeles.

Bonito oliva, A. (2002): Pollock, Giunti Gruppo Editoriale, Firenze.

Boulez, P. (1984): Puntos de referencia, Gedisa, Barcelona.

Brown, E. (1989): Unpublished interview by John Holzaepfel, Archives of the Earle Brown Music Foundation, New York.

CAGE, J. (1990): I-VI, Harvard University Press, Cambridge.

Cox, C.; Warner, D. (eds.) (2004): Audio Culture: Readings in Modern Music, Continuum International Publishing, New York.

De la CALle, R. (2007): «Sobre las relaciones entre música y pintura», AISTHESIS, 42: 87-97.

Dickinson, P. (1987): CageTalk: Dialogues with $\mathcal{E}$ about John Cage. Disponible en: http:/ / www.newmusicbox.org/articles/CageTalk-Earle-Brown-Interviewwith-Peter Dickinson/ [Consulta: 26/02/2021].

Duffie, B. (s. f.): Composer Earle Brown. A Conversation with Bruce Duffie. Disponible en: http:/ / www.bruceduffie.com/brown.html [Consulta: 26/02/2021].

Frank, E. (1983): Pollock, Abbeville Press, New York. 
GANN, K. (2006): Music Downtown: Writings from the Village Voice, University of California Press, Los Angeles.

GARCíA, M.S. (1996): «Las flores del infierno: Manolo Millares y Jackson Pollock», Anales de Historia del Arte, 6: 217-234.

Greenberg, C. (1948): «The Decline of Cubism», Partisan Review, 3: 366-369.

Guilbaut, S. (1990): De cómo Nueva York robó la idea de arte moderno, Mondadori, Madrid.

Hoek, D. J. (2004): «Documenting the International Avant Garde: Earle Brown and the Time-Mainstream Contemporary Sound Series», Notes, 61 (2): 350-360.

Holmes, T. (2005): Electronic and Experimental Music: Pioneers in Technology and Composition, Routledge, New York.

Hunter, S. (1958): Jackson Pollock 1912-1956, Whitechapel Art Gallery, London.

Jachec, N. (2011): Jackson Pollock. Obras, escritos, entrevistas, Polígrafa, Barcelona.

KIM-Conen, S. (2009): In the Blink of an Ear: Toward a Non-Cochlear Sonic Art, The Continuum International Publishing Group, New York.

KIM, R.Y. (ed.) (2017): Beyond Notation: The Music of Earle Brown. University of Michigan Press, Ann Arbor

KLeE, P. (1987): Diarios 1898-1918, Alianza Editorial, Madrid.

KozInN, A. (2007): «Picture a Pollock Painted by Harps», Music Review. Disponible en: http:/ / www.nytimes.com/2007/05/23/arts/music/23zank.html?_r=0 [Consulta: 26/02/2021].

MEYeRs, O. (2001): Mobility in the Works of Alexander Calder and Earle Brown, McGill University Press, Montreal.

Nyman, M. (1999): Experimental Music: Cage and Beyond, Cambridge University Press, Cambridge.

Nyman, M. (2006): Música experimental. De John Cage en adelante, Documenta Universitaria, Girona.

Pytheas Center For Contemporary Music (s. f.): Earle Brown (1926-2002). Disponible en: http:/ / www.pytheasmusic.org/brown_earle.html [Consulta: 26/02/2021].

Revill, D. (1992): The Roaring Silence: John Cage, A Life, Arcade Publishing, New York.

Rosenberg, H. (1952): «The American Action Painters», Art News, 51 (8): 22-50.

SAunders, F. (2013): The Cultural Cold War: The CIA and the World of Arts and Letters. New York Press, New York.

SIEGEL, J. (1999): Painting After Pollock. Structures of influence, OPA, New York.

Smigel, E. (2003): «The New York Schools of Music and Visual Arts», American Music, 21 (2): 249-254.

The Earle Brown Music Foundation (2013): Module I. Disponible en: http:// www.earle-brown.org/works/view/34 [Consulta: 26/02/2021].

Tomassoni, I. (1978): Pollock, Grosset \& Dunlap, New York.

Tomkins, C. (1968): The Bride and the Bachelors, Penguin, New York.

VALDEBenito, L.; CARRASCO, E. (2011): «Las relaciones entre la música y la pintura en el pensamiento de Theodor Adorno», Revista NEUMA, 1 (4): 44-57.

YÁÑEZ, P. (2006): «El amigo americano (80 años de Earle Brown)», Mundoclasico. com. Disponible en: https://www.mundoclasico.com/articulo/9642/Elamigo-americano-80-a\%C3\%B1os-de-Earle-Brown [Consulta: 26/02/2021]. 